\title{
THE THIRTY-SECOND ANNUAL MEETING OF THE SOCIETY
}

The thirty-second Annual Meeting of the American Mathematical Society was held in New York City, from Thursday to Saturday, December 31,1925-January 2, 1926. The regular sessions were held at Hunter College, January 1-2, preceded, on December 31, by the annual meeting of the Board of Trustees held at the Faculty Club of Columbia University. This was the first meeting of the Society in its history held in New York City at any other institution than Columbia University. It was made especially interesting by the exhibition at the College of an important part of the collection of objects bearing on the history of mathematics belonging to Professor D. E. Smith, of Columbia University, and by the invitation from Dr. G. A. Plimpton for attending members to view at his house his remarkable collection of old mathematical books and manuscripts. At the dinner on Friday evening, attended by about one hundred fifty members and their friends, Professor D. E. Smith presided; an attractive innovation was that of replacing most of the speeches by musical selections rendered by friends of the College, and by a one-act play called $A$ drama of relativity acted by the members of the Hunter College branch of the $\mathrm{Pi} \mathrm{Mu}$ Epsilon fraternity. At the close of the Saturday morning session it was voted to express the thanks of the Society to Hunter College and its department of mathematics, especially Professor Tomlinson Fort, for their cordial reception, to Dr. Plimpton for his hospitality, to Professor Smith for the loan of his collection, and to the musicians and students for making the dinner such an enjoyable occasion.

The attendance included the following one hundred twentynine members of the Society:

C. R. Adams, Alexander, W. L. Ayres, J. P. Ballantine, Belcher, Bell, S. R. Benedict, A. A. Bennett, Birkhoff, Bryan, Carruth, Carver, Abraham Cohen, L. W. Cohen, Dorweiler, Edmondson, Eisenhart, G. C. Evans, Everett, Feinler, Fiske, Fite, Fort, M. C. Foster, Philip Franklin, Frink, 
Gale, Gill, Glenn, P. H. Graham, M. C. Graustein, W. C. Graustein, Gravatt, Gray, Gronwall, C. C. Grove, Guggenbühl, Hammond, Hausle, Hawkes, Hedlund, Robert Henderson, Hille, Himwich, Hurwitz, Joffe, M. I. Johnson, Jonah, Karpinski, Kasner, Kazarinoff, O. D. Kellogg, B. F. Kimball, Koopman, Kormes, Kunte, Langer, Langman, Larew, Lefschetz, Littauer, Locke, McGiffert, MacColl, MacInnes, Maria, Mirick, H. H. Mitchell, Molina, L. T. Moore, Morenus, Richard Morris, D. S. Morse, Mullins, Oglesby, Pedersen, F. W. Perkins, Plimpton, Pogo, R. G. Putnam, Rainich, Ransom, Raynor, Reddick, R. G. D. Richardson, D. E. Richmond, Ritt, Rosenbaum, Schelkunoff, Seely, Seidlin, Siceloff, Silverman, Simons, C. E. Smith, D. E. Smith, W. M. Smith, Sosnow, Stark, Stocker, M. H. Stone, Swift, Tamarkin, T. Y. Thomas, Tracey, Tyler, Upton, Vandiver, Veblen, Waddell, Evelyn Walker, G. W. Walker, C. M. Walsh, Wedderburn, Weida, M. E. Wells, Wetzel, Anna Pell Wheeler, H. S. White, Whited, E. E. Whitford, Widder, Wiener, Wiggin, K. P. Williams, W. L. G. Williams, A. H. Wilson, R. G. Wood, M. M. Young.

The Secretary announced the election of the following persons to membership in the Society:

Mr. William Lord Bliss, United States Light and Heat Corporation, Niagara Falls;

Mr. Samuel Northrup Castle, advisory engineer, New York City;

Professor Robert Wood Clack, Alma College;

Professor William Erskine Cleland, Geneva College;

Mr. Leon Warren Cohen, Columbia University;

Professor Wilbur Haverfield Cramblet, Bethany College;

Mr. Ben Dushnik, University of Michigan;

Mr. Erwin Ulric Frey, designing engineer, New York City;

Dr. Leonard Franklin Fuller, vice-president, R. E. Thompson Manufacturing Company, Jersey City;

Mr. George Gibbs, consulting engineer, New York City;

Mr. Joseph D. Grant, University of Michigan;

Mr. Rollin Burke Hargrove, Southside High School, Elmira;

Mr. Gustav Arnold Hedlund, Hunter College;

Professor Edmund John Hirschler, Bluffton College;

Professor John Albert Hyden, Vanderbilt University;

Professor Florencio D. Jaime, Instituto Nacional Profesorado, Buenos Aires;

Professor Vladimir Karapetoff, Cornell University;

Mr. Spofford H. Kimball, University of Pittsburgh;

Mr. Mark Kormes, New York City;

Dr. Cooper Harold Langford, Harvard University;

Professor Charles Arthur Lindemann, Bucknell University;

Mr. Sebastian Barkann Littauer, Boys' High School, Brooklyn;

Professor Cristóbal de Losada y Puga, University of Lima;

President Frank Robert McBerty, North Electric Manufacturing Company, Galion, Ohio; 
Professor William Roberts McDaniel, Western Maryland College;

Professor C. Edward Magnusson, University of Washington;

Professor Thomas Adams Martin, Berea College;

Professor John Frederick Messick, Emory University;

Professor José Dionisio Morales, University of Porto Rico;

Professor Frank O'Callaghan, College of New Rochelle;

Mr. Boyd Crumrine Patterson, Johns Hopkins University;

Mr. A. Henry Pikler, Teudloff-Dittrich Manufacturing Company, Budapest;

Mr. Alexander Pogo, New York City;

Mr. William Henry Powell, Allis Chalmers Manufacturing Company, Milwaukee;

Miss Mary Josephine Quigley, Teachers College of the City of Boston;

Mr. Donald Everett Richmond, Brown University;

Mr. Vladimir Borisovich Rojansky, University of Oregon;

Mr. Frederick O. Runyon, consulting engineer, Newark;

Professor Charles Andrew Rupp, University of Texas;

Mr. Giulio G. Schroeder, English Electric Company, London;

Professor Alexander Smouroff, Electrotechnical Institute, Leningrad; Mr. Howard Parker Stabler, Harvard University;

Miss Marion Elizabeth Stark, Wellesley College;

Miss Ethel Clayton Stokes, Coker College;

Professor Charles Jacob Stowell, McKendree College;

President Frederick K. Vreeland, Vreeland Corporation, New York City;

Mr. C. A. M. Weber, Westinghouse Company, Springfield;

Mr. Edward Jones Willis, consulting engineer, Richmond;

Miss Frances May Wright, University of Oklahoma.

The Secretary announced that three of the nominees on the Allyn and Bacon sustaining membership had been transferred to ordinary membership and that the three following persons had on recommendation of the Secretary been elected in their place:

Miss Mildred E. Carlen, Connecticut College for Women;

Mr. C. M. Huber, University of Illinois;

Mr. Thomas W. Moore, Yale University.

The ordinary membership in the Society is now 1542, including 181 nominees of sustaining members and 81 life members. There are also 37 sustaining members, the total amount of whose annual dues is $\$ 5300$. The total attendance of members at all meetings, including sectional meetings, during the past year was 702; the number of papers read was 315. The number of members attending at least one meeting was 464 . At the annual election 254 votes were cast. 
The reports of the Treasurer and the auditors (Mr. S. A. Joffe and Professor Tomlinson Fort) were received, showing a balance of $\$ 14,070.72$, exclusive of special funds. Of this, $\$ 5568.43$ is reserved to secure the life memberships, and $\$ 3017.94$ for publications (including colloquium lectures) not yet completed; the appropriation of $\$ 3100$ made by the National Academy of Sciences for transferring the printing of our journals to this country is also included. Sales of the Society's publications during the year amounted to $\$ 4403.03$.

The trustees adopted a budget for 1926 showing estimated receipts and expenditures of $\$ 23,987.94$ and $\$ 23,962.94$ respectively; these receipts include the reserve for publications not yet completed and the National Academy appropriation, carried forward from 1925.

The Librarian reported that the Library of the Society now contains 6931 volumes. A catalogue has been prepared, and has been distributed.

At the annual election, which closed on Friday afternoon, the following officers and other members of the Council were chosen:

Vice-Presidents, Professors E. T. Bell and C. N. Moore.

Secretary, Professor R. G. D. Richardson.

Treasurer, Professor W. B. Fite.

Member of the Editorial Committee of the Bulletin, Professor W. R. Longley.

Member of the Editorial Committee of the Transactions, Professor Dunham Jackson.

Members of the Council, Professor Tomlinson Fort, Olive C. Hazlett, J. F. Ritt, E. B. Stouffer, and Norbert Wiener.

The telless appointed by President Birkhoff to count the ballots were Professors C. R. Adams and Lao G. Simons.

It was announced that President Birkhoff had appointed as representatives of the Society: at the inauguration of Charles Christopher Mierow as president of Colorado College on December 5, 1925, Professor C. H. Sisam; at the inauguration of Parke Rexford Kolbe as president of The Polytechnic Institute of Brooklyn on January 13, 1926, Professor Tomlin- 
son Fort; and that he had appointed Professors R. D. Bohannan (chairman), Arnold Dresden, C. N. Moore, S. E. Rasor and F. B. Wiley a committee on arrangements for the Summer Meeting of 1926.

At the meeting of the Council, Professor Virgil Snyder was named to succeed Professor E. V. Huntington as representative of the Society on the National Research Council for a period of three years beginning July 1, 1926. Professor E. S. Crawley and the Secretary were appointed to represent the Society on the Council of the American Association for the Advancement of Science.

To signalize the completion of the Endowment Campaign, a minute reviewing the events of the three years of effort was spread on the records of the Council and letters of felicitation and thanks were sent to Professors J. L. Coolidge and Oswald Veblen. To inform the members and other contributors concerning the present status of the affairs of the Society, it was decided to print the report of the Secretary for the years 1921-25. This report will appear in an early number of this Bulletin.

In order to mark the approaching retirement from academic life of Professor D. E. Smith, for nineteen years Librarian of the Society, a resolution of felicitation was adopted by the Council and read at the dinner.

A committee, consisting of Professors O. D. Kellogg (chairman), H. E. Hawkes, and H. H. Mitchell, was appointed to nominate the next incumbent of the Josiah Willard Gibbs Lectureship. This lecture will be given in connection with the Annual Meeting of 1926 in Philadelphia.

Professors E. T. Bell and A. J. Pell Wheeler were invited to give the lectures at the Madison Colloquium in 1927. It was announced that in the future all phases of the Colloquia and all book publications of the Society are to be entrusted to a committee of three, analogous to the Editorial Committee of the Transactions. This committee is to establish policies and procedure and to recommend to the Council action on all major matters. To inaugurate the reorganized procedure, the 
Council appointed as a committee Professors G. D. Birkhoff, G. A. Bliss and Oswald Veblen (chairman).

At the request of the Committee on Programme, Professor D. E. Smith delivered at the beginning of the Friday afternoon session an address on Material for the study of the history of mathematics, and Professor O. D. Kellogg at the beginning of the Saturday morning session an address on The Dirichlet problem.

President Birkhoff presided at the Friday morning session, and, relieved by Vice-President Evans, at the Saturday morning session. Retiring Vice-President Wedderburn presided on Friday afternoon, and Ex-President Veblen, relieved by Professor Kellogg, on Saturday afternoon.

Titles and abstracts of the papers, other than invitation addresses, read at this meeting follow below. The last five papers by Bell, and those by Fields, Koopman (second paper), Langford, Michal, Poor, Tamarkin and C. E. Wilder, J. M. Thomas, T. Y. Thomas, and R. L. Wilder were read by title.

1. Professor J. D. Tamarkin: Integro-differential equations and infinite determinants.

In this paper, the notion of the Green's function of a linear integrodifferential equation is introduced, and its expression in the form of an infinite determinant is given. This expression is used for the discussion of the existence of characteristic values and of the expansion problem of an arbitrary function in series of fundamental functions.

2. Professors J. D. Tamarkin and C. E. Wilder: Generalization of the notion of the adjoint problem in the theory of ordinary linear differential equations.

When the boundary conditions are of the end-point type, the adjoint problem has been discussed by Birkhoff and others. If the more general conditions involving the Stieltjes integral are used, the adjoint problem in the ordinary sense does not exist. It is the purpose of this paper to extend the notion to these general conditions.

3. Professor R. E. Langer and Mrs. Eleanor P. Brown: On the theory of integral equations with discontinuous kernels.

The subject of this paper is the integral equation

$$
u(x)=\lambda \int_{a}^{0} K(x, \xi) u(\xi) d \xi \text {, }
$$

in which the kernel is continuous except for a finite non-vanishing dis- 
continuity along the line $\xi=\chi$. The equation is reduced to a normal form, and is thereupon transformed into a system composed of an integrodifferential equation and boundary conditions. Under appropriate restrictions, characteristic values for the parameter $\lambda$ and corresponding characteristic solutions $u(x)$ are proved to exist, and their asymptotic forms are obtained. The set of characteristic solutions is proved to be closed. Lastly it is shown that the Fourier expansion of an arbitrary function in terms of the solutions of the given integral equation behaves essentially like the corresponding expansion in terms of the solutions of a related differential system. The discussion of the integral equation in which the kernel is continuous but has discontinuous derivatives will be presented shortly in a paper by the authors.

4. Professor Anna Pell Wheeler: Linear ordinary differential equations of the second order.

This paper deals with the expansion of arbitrary functions in terms of the characteristic functions of a self-adjoint differential equation with boundary conditions. The method used applies to the polar case as well as to the orthogonal case.

5. Professor W. B. Fite: Periodic solutions of linear differential equations.

In this paper the author considers the conditions under which a linear differential equation whose coefficients are all of period $l$ has one or more solutions of this period. Some simple relations connecting such solutions with the coefficients are derived.

\section{Professor Tomlinson Fort: The Fourier-Budan theorem} and mixed differential-difference equations.

In this paper a simple graphical analysis is given of the Fourier-Budan theorem. Then, observing that the set of functions used in that theorem are the solutions of the mixed equation $y_{n+1}+y_{n}{ }^{\prime}$, the author studies other mixed equations along lines suggested by the Fourier-Budan theorem.

7. Professor James McGiffert: Recurrence relations between solutions of the linear differential equation $d^{2} y / d x^{2}+((m-1) / x)$ $(d y / d x)=0, m$ being a positive integer.

If $y$ is a particular solution of the above equation for $m=m_{0}$, then $y=(1 / x)(d y / d x) m_{0}$ will be a solution for $m=m_{0}+2$. The proof follows a simple substitution. In particular, when $m=1$ the equation reduces to $\left(d^{2} y / d x^{2}\right)-y=0$, whose particular solutions are known to be $y=\cosh x$, and $y=\sinh x$. Hence, for $m$ odd, we have, as the particular solution, $y=((1 / x)(d / d x))^{(m-1) / 2} \cosh x$, or $y=((1 / x)(d / d x))^{m-1} \sinh x$. Thus we may obtain the particular, and hence the general, solution in closed form for all odd integral values of $m$. If $m=2$, the equation reduces to 
$\left(d^{2} y / d x^{2}\right)+(1 / x)(d y / d x)-y=0$, a particular solution of which is $y=I_{0}(x)$, where $I_{0}(x)$ is the Bessel function of zeroth order, with pure imaginary argument. Hence for all even values of $m$, we have the particular set of solutions $y=((1 / x)(d / d x))^{(m-2) / 2} I_{0}(x)$. Applications are varied.

8. Professor F. M. Weida: On the correlation between two functions.

This paper determines the correlation between two variates $X$ and $Y$, where $X$ and $Y$ are integral rational functions of $t$ of degrees $n$ and $m$ respectively, with real coefficients $a_{i}$ and $b_{j}$, defined for all values $t_{1}$ of $t$ on the range $c \leqq t_{1} \leqq d$. Rider* and Rietz ${ }^{+}$have investigated similar problems with different hypotheses. In the present paper it is shown that the coefficient of correlation $r$ (product moment coefficient) is given by

$r=(1 /(d-c)) \Sigma_{s=0}^{n+m}\left(\lambda_{s} /(s+1)\right)\left(d^{s+1}-c^{s+1}\right),(s=0,1,2, \ldots, n+m)$, where the $\lambda_{s}$ are functions of the $a_{i}$ and $b_{j}$. In the special case where $n=m=1, r=1$.

9. Professor M. C. Foster: Note on the envelope of a special family of spheres.

This paper considers the envelope of the two-parameter family of spheres with centers at the centers of geodesic curvature of these curves. Special orthogonal systems are considered. Use is made of the moving trihedral.

10. Dr. J. M. Thomas: On normal coordinates for the geometry of paths.

In this paper the author considers the transformations of normal coordinates brought about by a projective change of the affine connection. A necessary and sufficient condition for linear fractional transformation of the normal coordinates at every point is found to be the preservation of the symmetric part of the Ricci tensor. The paper also gives a set of identities connecting the covariant derivative of the $(r-1)$ th extension of any covariant vector with its $r$ th extension.

11. Dr. A. D. Michal: Functionals of $r$-dimensional spreads in $n$ dimensions admitting groups of point transformations.

In this paper a functional invariant theory is developed which includes as a special case the Poincare integral invariant theory. Existence theorems are given. Invariants of $r$-parameter groups and functionals attached to trajectories of differential equations are also considered. A certain modified tensor analysis is employed in the development of these invariant theories.

* American Mathematical Monthly, vol. 31 (1924), No. 5.

+ Quarterly Publications of the American Statistical Society, September, 1919, and Annals of Mathematics, (2), vol. 23, pp. 292-300. 
12. Dr. A. D. Michal: Concerning certain solvable equations with functional derivatives.

This paper is concerned with types of functional equations with functional derivatives which have as solutions certain functionals whose interesting properties are analogous to those of the Appell polynomials. In certain special cases these functional theories are isomorphic with the ordinary Appell polynomial theory. Such, for example, is the case in Evans' functional algebra when functional differentiation is defined in a suitable way. The equations in question involve, in general, functional derivatives of order higher than one as well as of order one.

\section{Professor J. C. Fields: Representation of the branches of} an algebraic function of several variables.

The representation of the branches of an algebraic function of several variables given in the present paper was, essentially, obtained by the author in the summer of 1903; it is this representation to which reference is made in the preface to the author's book Theory of the Algebraic Functions of a Complex Variable (Berlin, 1906). Presentation to the Society and publication have been postponed until the present time in the hopes of obtaining precise results for points on what is here called the restricted singular manifold.

14. Professor J. C. Fields: The existence theorem for the branches of an algebraic function of a complex variable.

Let $(i) f(x, u)=0$ be an irreducible integral algebraic equation and suppose this equation to be satisfied by a pair of values $z=a, u=b$. Expressing the left member in terms of powers of $(z-a)$ and $(u-b)$, and arranging according to dimensions, the equation can be written in the form (ii) $u_{n}+u_{n-1}+\cdots+u_{r}=0$, where $r \geqq 1$. If $r=1$, this equation is satisfied by a relation of the form (iii) $u-b=P\left((z-a)^{1 / \nu}\right)$, where the right member is a series in ascending integral powers of $(z-a)^{1 / \nu}$, and we may have $\nu=1$. We prove that also when $r>1$ the equation (ii) is satisfied by a relation of the type (iii).

\section{Professor R. L. Wilder: On the definition of simple closed curve.}

All the definitions that have been given of a simple closed curve $M$ require that $M$ be bounded or closed or both. The following definition requires neither of these conditions: A simple closed curve $M$ is a quasiclosed curve which is connected im kleinen. (A quasi-closed curve is the sum of two sets $M_{1}$ and $M_{2}$ which are irreducibly connected, respectively, between two points $A$ and $B$, and such that $M_{1}-(A+B)$ and $M_{2}-(A+B)$ are mutually separated.) It is also pointed out that a simple closed curve may be defined as a connected im kleinen continuum which is the sum of two sets $M_{1}$ and $M_{2}$ irreducibly connected between two points $A$ and $B$, such that $M_{1} \times M_{2}=A+B$. 
16. Dr. C. H. Langford: On a type of completeness characterizing the general laws for separation of point pairs.

This paper is based on Huntington's sets of postulates for separation of point pairs. The class of general laws is the class of all first-order propositions which are affected by the single applicative "every," or by the single applicative "some" introduced hypothetically. Huntington's sets are formulated on the base $K, R_{\mathbf{4}}$. Any such set is "complete" if it selects one or the other of every pair of mutually contradictory general laws which can be constructed on the given base. It is shown that any one of these sets has this property. Any first-order proposition in $n$ variables, formulated in terms of the base $K, R_{4}$ and involving a single use of "every," or, if the proposition be hypothetical, possibly a single use of "some," follows from each of the sets or else its contradictory follows.

17. Professor Evelyn Walker: Methods of tangents in the seventeenth century.

The problem of finding a general method of tangents became prominent and was solved in various ways during the seventeenth century. Fermat and Roberval had succeeded in finding such methods by 1636 , as is shown by their correspondence in that year. Fermat's method was an analytic one which he called that by maxima and minima, but which is almost identical with the modern method of approach through Cauchy's fraction. Roberval had two methods: an analytic one, now lost, and a mechanical one founded upon composition of motions. The latter was discovered independently, a little later, by Torricelli. Descartes published an analytic method in 1637 , and gave a full description of a mechanical one in 1638. Barrow's two methods differed little from those of Fermat and Roberval, while those of Wallis were identical with them. Newton's method of fuxions is an analytic combination of the two, and his mechanical method, using the instantaneous center of rotation, is precisely the second one discovered by Descartes. Leibniz's solution of the problem added only a new symbolism.

18. Professor E. T. Bell: The problem of general arithmetic. Preliminary report.

The problem is to express in abstract form the existing theory of numbers, and from the abstract set of relations thus obtained to derive all sets of entities satisfying the relations. For example, there is an arithmetical theory of classes, in which there is a unique factorization theorem, a theory of class congruences, a theory of class forms, etc. Division in this theory is not unique, nevertheless it is possible to define prime classes and unique factorization. This is an example of solving the set of abstract relations. Others are mentioned.

19. Professor E. T. Bell: On class number relations implied by representations as sums of an odd number of squares. 
A complete set (about 200) of the above-mentioned relations is obtained. The set is defined by the condition that any member of it shall involve, in addition to the class number functions, only algebraic functions of divisors. For $3,5,7,9,11$ or 13 squares, such results exist, ceasing permanently with the case of 15 squares.

20. Professor E. T. Bell: On the arithmetical applications of the power series, for elliptic functions.

In this paper, there is developed for the first time a fairly general method for eliciting from the power series for doubly or multiply periodic functions the arithmetical information contained in them. This is of a different nature from the classical applications of elliptic functions to the theory of numbers, and cannot be briefly abstracted.

\section{Professor E. T. Bell: Commutative algebraic inversions.}

By means of a simple algebra of the multiplicative symbolic properties of symmetric functions, the general inversions of $\mathrm{H}$. F. Baker* and L. Gegenbauer ${ }^{+}$are generalized, and the proofs considerably simplified.

22. Professor E. T. Bell: Periodic functions of $n$ variables in an algebraic number field of degree $n$.

The functions considered generalize to any algebraic number field the functions for the field generated by a cube root of unity, of Olivier, Hellwig, Glaisher, Nicodemi and Appell. A new type also is investigated. One set is periodic in $n$ variables, the periods depending upon the ground-number of the field; all have algebraic addition theorems. The derivatives "recur" in a novel way. The tensor algebra is used to shorten computations.

23. Professor E. T. Bell: A ray of numerical functions of $r$ arguments.

This paper will appear in full in an early issue of this Bulletin.

24. Professor H. S. Vandiver: Application of the theory of relative cyclic fields to both cases of Fermat's last theorem. Second paper.

In this paper several theorems are obtained, including the following: If $x^{p}+y^{p}+z^{p}=0$ is satisfied in integers none zero and each prime to the odd prime $p$, then $\left(\left(n^{p-1}-1\right) / p\right) D_{0} \equiv 0,\left(\left(n^{p-1}-1\right) / p\right) B_{(s+1) / 2} D_{8} \equiv 0(\bmod p)$, $s=1,3, \cdots, p-4 . I\left(\alpha^{d}-\beta^{a}\right)$ is defined by $\{\theta / \mathfrak{p}\}=\alpha^{I(\theta)}, \theta=\alpha^{a}-\beta^{d} ; \mathfrak{p}$ is a prime ideal divisor of the ideal $(n), n$ being a rational odd integer $\neq 0$ or 1 $(\bmod p) ; \alpha=e^{2 i \pi / p}, \beta=e_{2 i \pi /(n-1)} ; a$ is some integer in the set $1,2, \cdots, n-2$,

* Proceedings of the London Society, vol. 21 (1889), pp. 30-32.

+ Wiener Sitzungsberichte, vol. 102 (1893), part 2, pp. 951-978. 
other than $(n-1) / 2$; the $B$ 's are the numbers of Bernoulli, $B_{1}=1 / 6$, $B_{2}=1 / 30$, etc., and $\{\theta / \mathfrak{p}\}$ is defined as the power of $\alpha$ such that $\theta^{N(p)-1 / p} \equiv$ $\{\theta / \mathfrak{p}\}(\bmod \mathfrak{p}), N(\mathfrak{p})$ being the norm of $\mathfrak{p}$.

\section{Mr. G. Y. Rainich: Remark on paraphrases.}

A function $F$ is given by a power series; a linear relation with constant coefficients holds among $m$ values of $F$ obtained by multiplying the arguments by $m$ sets of $n$ constants; then the same relation holds for any other power series whose coefficients corresponding to zero coefficients of $F$ vanish. In particular, $F$ may be a general function which acquires a constant factor when some of its arguments are multiplied by an $r$ th root of unity; then $f$ is any function of the same description. For $r=2$, we have the case used by E. T. Bell in his Paraphrases.

26. Mr. Orrin Frink: On the representation of Boolean algebras as linear algebras.

It is assumed in the literature that Boolean algebras cannot be represented as linear algebras. In this paper, it is shown that all Boolean algebras, except certain infinite ones not capable of interpretation in terms of sets, can be so represented. The Boolean algebras are represented as semi-simple commutative linear algebras, the field of scalars being $G F(2)$. By taking other fields we get generalizations of Boolean algebras. A representation in terms of matrices is also given, the elements of the algebra being matrices in the normal form, reduced modulo 2. The addition operation in these representations is not the usual logical addition, but the alternative operation $x y^{\prime}+x^{\prime} y$ called "addition modulo 2." A set of rules for Boolean algebras when this operation replaces logical addition in the fundamental operations is given.

\section{Mr. F. J. Feinler: Recurrence formulas for the Bernoulli} numbers derived from zero differences.

In the present paper, proofs and formulas omitted from, one of the author's earlier papers* are given.

28. Mr. F. J. Feinler: Zero differences and oscillating prime numbers.

It is shown that certain derived zero differences consist of peculiar sequences of factorials multiplied by an arithmetical series of the $n$th order. The investigation includes the thirteenth order of derived zero differences illustrating the phenomenon of oscillation for the prime numbers $3,5,7$, $11,13$.

* On the Bernoulli numbers, read before the San Francisco Section of the Society on December 22, 1923, and published in the Messenger of Mathematics, vol. 55 , p. 40. 


\section{Professor L. C. Karpinski: Generalization of a theorem of Nicomachus.}

In the series of odd numbers the first one gives $1^{3}$, the next two $2^{3}$, the following three give $3^{3}$, and so on. In the present paper, the author extends the theorem to fifth and seventh powers, and gives further a generalization of which the theorem of Nicomachus is a special case. This theorem is that in the series of odd numbers, if we omit $((n-1) / 2) n^{k}$ odd numbers, the sum of the following $n^{k}$ odd numbers will be $n^{2 k+1}$. A further generalization of this theorem has been made by Mr. Norman Anning and Mr. E. B. Escott.

30. Dr. T. H. Gronwall: On the region of convergence of the power series expansion of a harmonic function of three variables.

Let $U(x, y, z)$ be harmonic inside the sphere $x^{2}+y^{2}+z^{2}=1$; then the expansion of $U$ in powers of $x, y$ and $z$ will converge when $|x|,|y|$ and $|z|$ are interior to the convex solid $S$ obtained by capping the sphere $|x|^{2}+|y|^{2}$ $+|z|^{2}=\frac{1}{2}$ with its three tangent cones from $(1,0,0),(0,1,0)$ and $(0,0,1)$ as vertices. Conversely, if $\left|x_{0}\right|,\left|y_{0}\right|,\left|z_{0}\right|$ is any point on the boundary of $S$, then $\xi, \eta$ and $\zeta$ may be determined on the unit sphere in such a manner that $1 /\left[(x-\xi)^{2}+(y-\eta)^{2}+(z-\zeta)^{2}\right]^{1 / 2}$, which is harmonic inside the unit sphere, will diverge for some complex values $x_{1}, y_{1}, z_{1}$ of the variables such that $\left|x_{1}\right|=\left|x_{0}\right|,\left|\dot{y}_{1}\right|=\left|y_{0}\right|,\left|z_{1}\right|=\left|z_{0}\right|$.

31. Dr. T. H. Gronwall: A differential equation in the DebyeHückel theory of strong electrolyles.

Let $F(\psi)$ increase monotonely from $-\infty$ to $+\infty$ as $\psi$ increases from $-\infty$ to $+\infty$ and consider the differential equation $d^{2} \psi / d r^{2}+(2 / r)(d \psi / d r)=$ $F(\psi)$. The qualitative properties of all solutions are investigated, and used to prove existence and uniqueness of a solution with the boundary conditions $d \psi / d r=c(<0)$ at $r=a(>0)$ and $\psi \rightarrow 0$ as $r \rightarrow \infty$, by a method involving an apparently novel application of the Dedekind cut. The results are applied to the numerical integration of the case $F(\psi)=\Sigma_{1}^{n} \alpha_{\nu} \beta_{\nu} e^{\beta \nu \psi}$, where all $\alpha_{\nu}>0$ and $\Sigma_{1}^{n} \alpha_{\nu} \beta_{\nu}=0$, upon which the Debye-Hückel theory is founded.

32. Mr. F. W. Perkins: A function related to Poisson's integral.

In the first section of this paper, it is shown that the unique function $u(P)$, finite and harmonic in a half plane, and taking on boundary values $f(\pi)$ on the line $L$, is the "apparent average" of the values $f(\pi)$ as seen from $P$, that is, $u(P)=(1 / \pi) \equiv \int_{-\pi / 2}^{\pi / 2} f(\pi) d \omega$, where $\omega$ is the angle from the perpendicular on $L$ through $P$ to the ray $P \pi$. The remainder of the paper is devoted to applications of this result in the study of certain properties of Poisson's integral. 


\section{Mr. F. W. Perkins: On the oscillation of harmonic functions. Second paper.}

Problems similar to those considered in an earlier paper of the same title presented to this Society on December 30, 1924, are discussed. Properties of functions finite and harmonic in a half plane, corresponding to those previously treated for the circle, are established. Some theorems on the oscillation of harmonic functions of three variables are proved, in particular, an analogue of a theorem due to Neumann concerning the oscillation of a harmonic function on a pair of concentric circles.

34. Dr. B. O. Koopman: On rejection to infinity and exterior motion in the restricted problem of three bodies.

The restricted problem of three bodies has been studied in detail only when the particle is in the neighborhood of points of equilibrium or in the interior of an oval of zero velocity. In none of these cases does the question of rejection to infinity play any rôle. It is to this phenomenon, and types of motion in which its possibility is an essential feature, that the present paper is devoted. First we investigate individual half-orbits extending to infinity, find a criterion for their existence, and prove that they approach an asymptote. Next we investigate infinity from the point of view of a singularity of the equations of motion, and show how to reduce it to a well known form. Then we consider motion outside a closed oval of zero velocity containing the massive bodies, and extend to this case most of the results concerning the existence of the surface of section and symmetric periodic orbits obtained by Birkhoff* for the interior case. We end by studying the relative distribution of stable and unstable motions.

\section{Dr. B. O. Koopman: Flow in a Möbius strip.}

Let a Möbius strip be constructed from a rectangle of tin foil, and let the poles of a battery be placed in contact with it: what is the nature of the flow of electricity in the strip? A simple method is developed for solving this problem, and establishing the uniqueness of the solution. A typical case is treated in detail, and compared with the corresponding problem for the cylinder; it is found that there is a singularity in the former case which does not occur in the latter.

\section{Professor Philip Franklin: A geometric characterization} of equipotential and stream lines.

The object of this paper is to find out when a harmonic function exists having a given family of analytic curves as its equipotential lines. The solution is obtained by constructing certain point functions depending intrinsically on the given curves, which are constant along these curves when

* The restricted problem of three bodies, Rendiconti di Palermo, vol. 39 (1915), pp. 1-70. 
and only when the harmonic function exists. A similar discussion is given for the characterization of the stream lines of a steady, two-dimensional fluid motion.

\section{Dr. T. Y. Thomas: On conformal geometry.}

Conformal geometry is presented as the invariant theory of the equations of transformation of a fundamental conformal tensor $G_{\alpha \beta}$ and by means of these equations the fundamental problem of the algebraic characterization of the conformal manifold is solved. The importance of the elimination equation by which second derivatives of transformations relating manifolds are given in terms of first derivatives is emphasized in establishing this characterization. Two types of elimination equations are given, the first of which is connected with $(n+1)$-dimensional manifolds, the second with $(n+2)$-dimensional manifolds.

38. Professors Max Born and Norbert Wiener: $A$ new formulation of the Heysenberg method of quantization.

The authors apply the theory of the harmonic analysis of non-periodic functions to the generalization to systems with continuous sequences of quantized states of Heysenberg's formulation of the quantum theory in terms of matrices.

39. Mr. G. Y. Rainich: Electricity and mass in certain static spaces.

Space-time is studied which corresponds to a static field with the further restriction that the direction of the gravitational force vector is a principal direction of both the Riemann tensor of the space and the mass tensor, the two other principal directions being indeterminate.

40. Miss Marion E. Stark: A self-adjoint boundary value problem associated with a problem of the calculus of variations.

The problem of this paper is that of finding among the arcs $y_{i}=y_{i}(x)$ $\left(i=1, \cdots, n ; x_{1} \leqq x \leqq x_{2}\right)$ having end points $\left(x_{1}, y_{11}, \cdots, y_{n 1}\right)$ and $\left(x_{2}, y_{12}, \cdots, y_{n 2}\right)$ that satisfy a set of equations of the form $\psi_{\mu}\left(x_{1}, y_{11}, \cdots\right.$, $\left.y_{n 1} ; x_{2}, y_{12}, \cdots, y_{n 2}\right)=0(\mu=1, \cdots, p \leqq 2 n+2)$ one which minimizes the integral

$$
I=\int_{x_{1}}^{x_{2}} f\left(x, y_{1}, \cdots, y_{n}, y_{1}^{\prime}, \cdots, y_{n}^{\prime}\right) d x .
$$

Let the desired minimizing arc be called $E_{12}$. After the statement of certain hypotheses and definitions, a useful theorem is proved involving a oneparameter family of arcs having a special set of variations along $E_{12}$. Then the first and second variations of the integral $I$ are found, together with the Euler equations and transversality conditions. A minimum problem is now seen to be suggested by the second variation. As it is investigated, new boundary conditions are developed. The boundary value 
problem of the second variation is then formulated, and a new necessary condition is found for a minimum of the original problem. A special case of the boundary value problem may be shown to have the property of selfadjointness according to a definition of self-adjointness given by Bliss.

\section{Mr. D. E. Richmond: Geodesics on surfaces of genus} zero with knobs.

A knob will be defined as a finite portion of surface $K$ which is (a) bounded by a closed curve $C$ with continuously turning tangent; (b) capable of being put into one-to-one continuous correspondence with the interior and boundary points of a circle; (c) such that the geodesics tangent to the boundary $C$ lie in $K$ in the immediate neighborhood of the point of contact. On any regular surface of genus zero with a finite number of knobs, we consider a certain set of geodesics issuing from any point not on a knob. The results resemble those obtained by Hadamard for surfaces of negative curvature.

\section{Dr. M. H. Stone: Series of Legendre polynomials.}

The aim of this paper is to give an "elementary" treatment of the Legendre polynomials and developments in terms of them. So far as the author is aware, the discussions of series of Legendre polynomials published hitherto have striven for generality and are correspondingly difficult. Here the properties of the polynomials $P_{n}(x)$ are derived from the definition

$$
1 / \sqrt{1-2 x r+r^{2}}=\sum_{n=0}^{\infty} P_{n}(x) r^{n}, \quad-1 \leqq x \leqq+1,
$$

by standard operations with infinite series. The relation $\left|P_{n}(\cos \theta)\right| \leqq M /$ $\sqrt{n} \sin \theta, 0>\epsilon \leqq \theta \leqq \pi-\epsilon$, is found by the use of Abel's lemma. The convergence of expansions in terms of the functions $P_{n}(x)$ is then treated for the range $-1<x<+1$ in cases where the coefficients approach zero with $1 / n$ fairly rapidly. The integral which plays the part of Dirichlet's integral in this theory is also examined.

\section{Dr. Mark Kormes: On linear operations.}

In this paper continuity of a linear operation in a general sense is defined and also in a restricted sense. For spaces of a finite number of dimensions, from continuity in the general sense follows continuity in the restricted sense. This is not the case for spaces of an infinite number of dimensions. It is sufficient for a linear operation in a space of a denumerably infinite number of dimensions to be bounded in order that it be continuous. Affine linear representations of $R_{m}$ on $R_{n}$ are defined, and the existence of measurable and non-measurable $(L)$ linear representations is established. An example is given of a solution of $f(x-y)-f(x)-f(y)$ which is one-to-one and not measurable with respect to the square measure. 
44. Mr. W. L. Ayres: End-point homogeneity in continuous curves containing no simple closed curve.

This paper considers the following problem: Suppose that in a continuous curve $M$ containing no simple closed curve any two sets of $n$ end points are chosen. Under what conditions will there exist always (no matter what two sets are chosen) a one-to-one continuous transformation of $M$ into itself which carries the points of the first set into the corresponding points of the second set? The author proves that if $n>3$ it is necessary and sufficient that $M$ contain a point $P$ such that $M$ consists of a set of arcs from $P$ to the end points of $M$, no two of which have any point in common except $P$. An example is given to show that the condition is not necessary unless $n>3$. It is also proved that if a continuous transformation of a continuous curve containing no simple closed curve leaves all, except possibly three, of the end points invariant it leaves the branch points invariant also.

45. Professor V. C. Poor: On definitions and transformation theorems in the theory of the homography.

This paper exhibits the close correlation of homography theory with the elements of vector analysis. Simpler definitions coextensive with the more complicated current form are pointed out and several transformation theorems given.

\section{Professor V. C. Poor: On the double layer potential.}

This note contains an elementary proof of the convergence of the double layer potential.

R. G. D. RICHARDSON, Secretary of the Society. 